\title{
IMPORTÂNCIA DA HUMANIZAÇÃO NA ADESÃO DOS USUÁRIOS AOS SERVIÇOS DE SAÚDE NA ATENÇÃO PRIMÁRIA ${ }^{1}$
}

\author{
THE IMPORTANCE OF HUMANIZATION IN USERS' \\ ADHERENCE TO HEALTH SERVICES IN PRIMARY CARE
}

\author{
Adailson José Souza Santos Sobrinho² e Jailma de Frias Santos ${ }^{3}$
}

\section{RESUMO}

Este estudo teve como objetivo relatar a experiência de um cirurgião-dentista, residente de um programa multiprofissional, o qual atuou em uma Unidade de Saúde da Família adotando o cuidado humanizado como estratégia para adesão dos usuários aos serviços de saúde. A partir das experiências vivenciadas, foi observado que o atendimento humanizado melhorou a adesão dos assistidos nos serviços, estimulou a confiança e o vínculo entre os profissionais e usuários, proporcionando ambientes acolhedores e, como resultado, foi observada a longitudinalidade da atenção à saúde. Diante disso, a humanização nos serviços se mostra uma estratégia eficiente para o cuidado continuado, vinculação dos usuários e prestação de assistência de forma integral, garantindo a prática dos princípios e diretrizes do Sistema Único de Saúde.

Palavras-chave: Humanização, Atenção Primária à Saúde, Saúde Bucal.

\section{ABSTRACT}

This study aimed to report the experience of a dentist, resident of a multidisciplinary program, who worked at a Family Health Unit adopting humanized care as a strategy for users' adherence to health services. Based on the experiences, it was observed that humanized care improved the adhesion of those assisted to the services, stimulated trust and the bond between professionals and users, providing welcoming environments and, as a result, the longitudinality of health care was observed. Therefore, the humanization of services proves to be an efficient strategy for continued care, linking users and providing comprehensive care, ensuring the practice of the principles and guidelines of the Unified Health System.

Keywords: Humanization. Primary Health Care. Oral Health.

1 Relato de experiência.

2 Cirurgião - Dentista especialista em Saúde da Família. E-mail: adailsonsobrinho@hotmail.com

3 Cirurgiã - Dentista especialista em Saúde da Família. E-mail: jailma_friassantos@hotmail.com 


\section{INTRODUÇÃO}

A humanização na Atenção Primária à Saúde (APS), enquanto política pública, tem se afirmado na construção de espaços que alteram as formas de compreender e produzir saúde. Aspirando a desconstrução do modelo médico hegemônico, a oferta da atenção de maneira humanizada possibilita a compreensão dos processos que levam os indivíduos ao adoecimento, em busca da resolução de problemas e melhorias na qualidade da assistência. Além disso, a humanização na APS contribui para a construção do vínculo entre usuários e profissionais, algo fundamental para a continuidade do cuidado integral (ZUGE, 2012).

Os programas de residência multiprofissionais em saúde apostam no vínculo, através da humanização, por possibilitarem que as relações se estreitem e facilitem o tratamento e a adesão dos usuários aos serviços, além de promoverem a longitudinalidade do cuidado (BRASIL, 2008). Esses programas foram criados no ano de 2005 e regulamentados pela lei 11.129 e constituem formações de ensino de pós-graduação lato sensu com duração prevista de vinte e quatro meses. O objetivo é capacitar os profissionais para atuarem no SUS, dentro da Estratégia de Saúde da Família (ESF), de forma crítico-reflexiva, tomando como base o modelo de atenção de vigilância à saúde (BRASIL, 2012).

No primeiro ano, o pós-graduando atua na área assistencial em um território adscrito. Exerce a promoção da saúde, implementando estratégias para facilitação do acesso e do acolhimento dos usuários, busca o conhecimento do território no qual está inserido por meio dos equipamentos sociais e suas características, realizando, através disso, a atenção domiciliar, a educação em saúde e a saúde na escola. No segundo ano, atua nas redes de atenção e linhas de cuidado, elaborando, pactuando fluxos, realizando avaliação dos serviços de saúde, dentre outras funções (COSTA et al., 2009).

Devido ao caráter multiprofissional do programa, é possível despertar no residente a necessidade de inserção em diversos espaços de cuidado, propiciados pela dinâmica da saúde da família. Esta característica do trabalho multidisciplinar possibilita a ampliação da clínica, a capacidade inventiva do trabalhador, o apoio matricial, a elaboração de projetos terapêuticos singulares e a comunicação intersetorial. Não obstante, também contribui para a utilização de tecnologias leves como a humanização, um dos eixos fundamentais para a vinculação dos usuários aos serviços de saúde (FESF-SUS, 2018).

Desta forma, o objetivo deste artigo é relatar a experiência de um residente, cirurgião-dentista, que atuou em uma Unidade de Saúde da Família (USF), localizada em um município da Bahia e que adotou o cuidado humanizado como estratégia para adesão dos usuários aos serviços de saúde.

\section{MATERIAIS E MÉTODOS}

Para este artigo foram utilizados os registros das vivências do cirurgião-dentista no Ambiente Virtual de Aprendizagem (AVA) do programa de residência multiprofissional em saúde da família do 
qual fez parte, postados no período de março de 2019 a fevereiro de 2021. Foram consultados também arquivos do Ministério da Saúde e publicações nacionais nas plataformas de pesquisa da Scielo, Medline e Biblioteca Virtual de Saúde com os seguintes descritores: Humanização, Saúde Bucal e Atenção Primária à Saúde, Humanização, sendo selecionados os que mais se aproximavam do objetivo da escrita do texto, sendo excluídos aqueles com idioma inglês e que não se associavam à proposta do artigo. Ao todo foram selecionados 11 artigos em língua portuguesa.

\section{CENÁRIO DE PRÁTICAS}

A unidade de saúde da família, objeto deste relato, está localizada em um município baiano. Ele dispõe de uma rede de assistência composta por uma Unidade de Pronto Atendimento (UPA 24h), um Centro de Especialidades Odontológicas (CEO), um Centro de Atenção Psicossocial (CAPS), um hospital municipal e 15 Unidades de Saúde da Família (USF). Ainda compondo a rede de assistência social, há os centros de referência que contribuem para a condução de casos encaminhados (PREFEITURA MUNICIPAL DE DIAS D’ÁVILA, 2017).

Com uma população de aproximadamente 8.000 habitantes, o bairro em que a unidade está localizada é caracterizado como um território periférico, em constante crescimento, cuja população convive com vulnerabilidades sociais, como a falta de saneamento básico, segurança pública, infraestrutura, falta de oportunidade de trabalho e de lazer (SECRETARIA MUNICIPAL DE DIAS D’ÁVILA, 2017).

Objetivando melhorar a qualidade e o acesso da população aos serviços de saúde, estabelecer os valores do SUS no âmbito da atenção primária (AP) e proporcionar qualificação dos profissionais da rede, foi implementado em 2017 um programa de Residência Multiprofissional em Saúde da Família na USF. Esta residência é composta por uma equipe multiprofissional que contempla enfermeiros, cirurgiões-dentistas, fisioterapeutas, psicólogos, nutricionistas, profissionais de educação física que são residentes e uma equipe pedagógica formada por preceptores, apoiadores de núcleo e de campo (FESF-SUS, 2018).

Os preceptores acompanham a atuação dos residentes em serviço diariamente; os apoiadores de núcleo dão suporte aos preceptores e residentes semanalmente; e os apoiadores de campo atuam no acompanhamento das necessidades tanto dos preceptores, residentes e apoiadores de núcleo quanto dos trabalhadores que não compõem a equipe pedagógica do serviço.

Com a chegada da residência foi iniciado um processo de qualificação das ações de cuidado na USF, dos processos de trabalho, da educação permanente e da formação em saúde no serviço. A experiência do cirurgião-dentista foi iniciada em março de 2019, sendo este apresentado às demandas do serviço, dentre as que são sabidamente ofertadas na atenção primária, como o acolhimento à demanda espontânea, atendimentos odontológicos, atenção domiciliar e atividades coletivas. 


\section{ACOLHIMENTO}

Acolhimento é uma diretriz da Política Nacional de Humanização (PNH) que não tem local nem momento específico para o acontecimento, não exige um profissional capacitado exclusivamente para realizá-lo e deve fazer parte da prática de todos os trabalhadores dos serviços de saúde. Pode ser compreendido como parte do cuidado integral, articulando-se com o acesso e implementação de práticas de assistência nos serviços, em busca da resolubilidade e atendimento humanizado (BRASIL, 2008).

Para a prática de acolhimento é importante que o usuário se sinta à vontade para se expressar e ser ouvido pela equipe. Isto viabiliza um tratamento que mais se adeque à sua realidade, proporcionando maiores chances de adesão às terapêuticas propostas. Cabe ressaltar que em cada território essa prática se adapta à realidade local e deve considerar aspectos da comunidade, vulnerabilidades sociais, hábitos de vida, aspectos econômicos, entre outros (GUERRERO et al., 2013).

Nos momentos do acolhimento à demanda espontânea no serviço, o residente fazia o registro das necessidades de saúde dos usuários em um livro-ata destinado a esta finalidade. Além da identificação prévia do acolhido, eram também registrados o endereço, número identificador da família e agente de saúde, gênero, idade, sinais vitais e comorbidades preexistentes. Após ser avaliado, a partir da escuta sensível e qualificada, o acolhido era encaminhado para o profissional que melhor pudesse ofertar resolubilidade ao caso. Para isso era considerada também a classificação de risco, a fim de organizar a demanda de saúde, bem como proporcionar equidade (BRASIL, 2013).

Por ser cirurgião-dentista, por vezes, o residente acolhia casos de sua atuação profissional, como também ofertava resolubilidade daqueles que não eram de sua competência técnica, considerando para isso o limite da ética e do exercício das profissões da saúde. O mesmo ocorria com outros residentes de distintas categorias profissionais. Isto se deve aos momentos de educação permanente que foram ofertados na USF, que permitiram que todos os envolvidos no cuidado acolhessem casos para além de suas respectivas atuações específicas, propiciando a ampliação do trabalho em equipe e da intensificação do uso das tecnologias leves como estratégia de manejo em saúde (WARMLING et al., 2019).

No matriciamento realizado pelo dentista, enquanto recurso educativo, foram utilizadas imagens impressas e cartazes informativos, com linguagem acessível para os ouvintes que não tinham formação acadêmica em odontologia. Foram exibidos casos de saúde bucal mais comumente encontrados no cotidiano da USF, a fim de que fosse estimulado no acolhedor a possibilidade de detalhar a queixa do usuário e de que pudesse colher informações imprescindíveis para que o dentista realizasse a hipótese diagnóstica mais precisa possível (BRASIL, 2018).

Através do matriciamento, foi orientado também o fluxo para a condução das queixas odontológicas na unidade. Também foi criada a classificação de risco adaptada dos manuais de domínio público do Ministério da Saúde sobre acolhimento à demanda espontânea, conforme tabela 1 . 
Tabela 1 - Classificação de riscos odontológicos na USF Concórdia.

\begin{tabular}{|c|c|c|c|}
\hline $\begin{array}{c}\text { ATENDIMENTO } \\
\text { IMEDIATO - VERMELHO }\end{array}$ & $\begin{array}{c}\text { ATENDIMENTO } \\
\text { PRIORITÁRIO - AMARELO }\end{array}$ & $\begin{array}{l}\text { ATENDIMENTO NO DIA E } \\
\text { SEM PRIORIDADE - VERDE }\end{array}$ & $\begin{array}{c}\text { ATENDIMENTO DE CONSULTA } \\
\text { E ATIVIDADE - AZUL }\end{array}$ \\
\hline $\begin{array}{l}\text { Abcesso (quadro de algia, inchaço, } \\
\text { presença ou não de pus, trismo ou } \\
\text { febre, indicativo de celulite) }\end{array}$ & $\begin{array}{l}\text { Dor intensa e espontânea, } \\
\text { que não cessa com o uso de } \\
\text { analgésicos }\end{array}$ & $\begin{array}{l}\text { Dor provocada, que cessa com } \\
\text { retirada do estímulo }\end{array}$ & Avaliação bucal \\
\hline $\begin{array}{l}\text { Trauma dentário ocorrido até } 30 \\
\text { minutos (avulsão, luxação } \\
\text { extrusiva, luxação lateral) }\end{array}$ & $\begin{array}{l}\text { Complicações pós-cirúrgicas } \\
\text { (alveolite) }\end{array}$ & Remoção de sutura & Restauração fraturada sem algia \\
\hline $\begin{array}{l}\text { Laceração e hemorragias em } \\
\text { região de cabeça e pescoço }\end{array}$ & $\begin{array}{l}\text { Trauma dentário ocorrido entre } \\
30 \text { minutos e } 1 \text { hora }\end{array}$ & $\begin{array}{l}\text { Alterações da mucosa oral } \\
\text { (placas, bolhas, nódulos) }\end{array}$ & $\begin{array}{l}\text { Extração de decíduo ou } \\
\text { permanente }\end{array}$ \\
\hline Luxação da ATM* & $\begin{array}{l}\text { Restauração fraturada com } \\
\text { sintomas de algia associados }\end{array}$ & Sangramento gengival com algia & Sangramento gengival sem algia \\
\hline
\end{tabular}

*ATM= articulação temporomandibular.

Fonte: Autor (2020).

\section{ATENÇÃO DOMICILIAR}

A atenção domiciliar (AD) é caracterizada pelo conjunto de ações de promoção à saúde, prevenção, tratamento e reabilitação de doenças, prestadas em domicílio. Ela objetiva ampliar o acesso da população aos serviços, sendo ofertada a indivíduos e famílias que têm dificuldades para se deslocar até as USF, garantindo, desta maneira, a integralidade, a continuidade e a equidade do cuidado, além do estabelecimento e fortalecimento da criação de vínculos entre usuário e profissionais de saúde (RAJÃO; MARTINS, 2020).

As visitas domiciliares (VD) faziam parte da agenda de todos os residentes e ocorriam semanalmente, de acordo com a necessidade identificada pela equipe, pelos usuários e familiares. A identificação das demandas ocorria a partir das discussões de casos nas reuniões de equipe, nas quais cada caso era avaliado a partir da priorização realizada por meio de instrumento específico, que permitia a classificação quanto à necessidade de atenção à saúde. $\mathrm{O}$ instrumento consistia em uma escala que determinava o risco social e de saúde do indivíduo a partir de itens que, quando somados, tinham como resultado o potencial de adoecimento, sendo os escores 5-6 de baixo risco, 7-8 risco médio e acima de 9 risco alto (SAVASSI; LAGE; COELHO, 2012).

Esta modalidade de atenções em saúde abrange grupos como puérperas, pessoas com necessidades especiais, indivíduos acamados e domiciliados. No âmbito odontológico, são avaliados aspectos como a higiene oral, presença de alterações bucais e necessidade de intervenção clínica odontológica ou encaminhamento para serviço especializado. Ainda são avaliadas as condições de saúde geral do sujeito com a finalidade de identificar quais dificuldades este possui para lidar com sua condição de saúde, como por exemplo dificuldade em organizar os horários das medicações, dificuldades para conseguir materiais para curativo domiciliar ou dificuldade para aceitação da condição de saúde, além de identificar e monitorar outras condições, proporcionando o cuidado integral 
(BIZERRIL et al., 2015).

A abordagem dos outros componentes familiares é importante. O profissional identifica na dinâmica familiar a relação destes para com o indivíduo que está sendo abordado na visita, detectando possíveis falhas no manejo do cuidado e elaborando estratégias que visem a prevenção de agravos e promoção da saúde. Essa abordagem deve ser realizada pela equipe de forma multiprofissional, de maneira acolhedora, como aliada à melhora da condição observada e do fortalecimento do vínculo usuário/família com a equipe da atenção primária (BRASIL, 2012).

Ao longo de suas vivências, realizando visitas domiciliares, o dentista identificou que as condições de vida, os valores e os hábitos das pessoas precisavam ser compreendidos como distintos e que esses deviam ser entendidos de maneira singular. Observou atentamente os aspectos da vida cotidiana não só das pessoas passíveis de cuidado como da rede de apoio quando a mesma existia, atentando-se para a integralidade como princípio do SUS. Nos domicílios observou que ter prestado atenção para o cuidado ampliado em saúde também foi de importância significativa para a construção e manutenção de vínculos, fortalecendo o processo da humanização no ato de cuidar.

\section{ATENDIMENTOS ODONTOLÓGICOS}

Os atendimentos odontológicos também faziam parte da agenda do residente e aconteciam em turnos semanais. Eram organizados de maneira que fosse possível agendar usuários para primeira consulta programática, consulta de retorno e urgências.

Os pacientes de primeira consulta programática eram agendados após uma atividade coletiva mensal. Nesta, era realizada educação em saúde com tema previamente definido e eram avaliadas as necessidades bucais de cada indivíduo. Nesse momento a queixa principal do sujeito era acolhida e discutida com o mesmo a partir de abordagens cabíveis para cada caso. Ressalta-se que tal interação se dava de maneira objetiva, com a finalidade apenas de sanar dúvidas dos usuários quanto ao autocuidado, sendo possível, naquele momento, solicitar tomadas radiográficas para avaliação odontológica e prescrever medicamentos.

No consultório, dados como dentes cariados, dentes com indicação de exodontia ou endodontia, necessidade de realização de prótese total ou parcial, além de presença de comorbidades como hipertensão, diabetes mellitus ou síndromes eram registradas. Também era anotado o recebimento de auxílios governamentais, como o bolsa família, por exemplo, a fim de promover a equidade do cuidado com os mais vulneráveis (WARMLING et al., 2019).

Os dados eram então discutidos com o usuário, explicando-se de maneira simples e objetiva como seriam as condutas clínicas ao longo do tratamento. Concomitantemente a isso, o dentista buscava sempre atentar à fala e aos questionamentos do indivíduo frente às suas condições de saúde, para que este pudesse compreender o porquê de tais abordagens e de que maneira contribuiria para 
a melhora da sua saúde. Nestes momentos, o residente observou que ouvir os usuários, acolher suas queixas e definir possíveis encaminhamentos proporcionava a criação e manutenção de vínculos e possibilitava a autonomia destes na construção dos tratamentos odontológicos, incentivando-os a cuidar da saúde bucal.

A autonomia do usuário na construção do seu tratamento o faz compreender a necessidade e a importância de determinados manejos clínicos, como por exemplo, a necessidade de uma adequação do meio bucal ante a restauração de um elemento dentário ou ao controle de uma condição sistêmica para a realização de exodontias. A coparticipação e a corresponsabilização ativa no autocuidado para com os problemas de saúde possibilitava ainda a criação de vínculo e adesão dos sujeitos ao serviço de saúde (GUERRERO et al., 2013).

Foi notado pelo dentista que os pacientes, ao perceberem o progresso dos tratamentos, sentiam-se mais dispostos a praticar o autocuidado. Pacientes antes descompensados com as doenças crônicas passaram a ser mais vigilantes com a manutenção da saúde, visto que a saúde bucal estava sendo acompanhada através de uma relação com a equipe de forma acolhedora e respeitosa. Este aspecto do cuidado, humanizado e integral, fez com que os sujeitos se sentissem satisfeitos com o serviço, mesmo que por vezes, este cuidado fosse dificultado por algumas ocasiões de infraestrutura ou organização interna do trabalho.

\section{DESAFIOS NA PRESTAÇÃO DO CUIDADO HUMANIZADO}

Alguns fatores influenciam os processos de trabalho que, consequentemente, dificultam o atendimento humanizado. A carência e má gestão de recursos no SUS, algo que se arrasta ao longo da sua história, limita a ação dos profissionais dentro da $\mathrm{AB}$ e prejudica a assistência aos usuários (SALDIVA e VERAS, 2018). A unidade de saúde em que o residente atuava tinha problemas de infraestrutura, onde os espaços físicos improvisados, inadequados e em péssimo estado de manutenção impediam a privacidade dos atendimentos.

Além disso, a falta de insumos impossibilitava ou adiava a realização de procedimentos, dificultando o acesso aos serviços de saúde, levando os pacientes à insatisfação com as ofertas do serviço e sem estímulo para voltar a frequentar a USF. Isto repercutia não só em uma decisão individual, como também da comunidade, pois a informação sobre a qualidade dos serviços se propagava com facilidade na comunidade. Em um cenário como este, os profissionais se sentiam limitados e desmotivados. Somou-se a isto os casos onde havia sobrecarga de atividades e funções, que acabavam implicando em um desgaste físico e emocional, impactando diretamente na conduta acolhedora.

Outra dificuldade encontrada pelo residente foi a articulação com a rede de saúde. Por vezes, encaminhar o usuário para centros especializados como CEO ou CRAS era algo dificultado pelos processos burocráticos e alta demanda. Isto não significa exatamente que o cuidado humanizado 
fosse impedido de ser posto em prática, mas trazia dificuldades em manter o usuário motivado e, consequentemente, vinculado ao serviço de saúde.

A experiência vivenciada pelo cirurgião-dentista, mostrou que a alta demanda na busca por procedimentos reabilitadores, como próteses dentárias, fazia com que o indivíduo aguardasse meses para que fosse agendado para atendimento, gerando uma sensação de insatisfação, muitas vezes contornada pelo acolhimento das angústias e frustrações através do vínculo estabelecido do usuário com o profissional.

Além disso, a rotatividade do programa de residência se torna um fator determinante no processo de vinculação. A cada ano, novos residentes adentram ao programa e passam a assumir as agendas das suas respectivas equipes. Embora essa transição ocorra de maneira gradual, vincular o usuário com um novo profissional e uma nova equipe pode se mostrar uma tarefa que demanda paciência e cautela, pois o indivíduo, ao não se sentir acolhido, tende a se desmotivar e, consequentemente, desvincular-se das unidades.

\section{CONCLUSÃO}

A humanização, enquanto política pública de saúde, garante aos usuários, trabalhadores e aos gestores a efetivação dos princípios e diretrizes do SUS, bem como o exercício de processos de trabalho de forma adequada. Ofertar aos usuários a escuta qualificada viabiliza a construção de relações de confiança e estimula o vínculo entre usuários e trabalhadores. Isto pode mobilizar os atores envolvidos no cuidado para o pleito de melhorias na qualidade dos serviços prestados à população.

Os programas de residência, em especial os de Saúde da Família, qualificam profissionais por meio da formação de ensino em serviço fundamentados no modelo de atenção de vigilância à saúde, proporcionando uma especialização pautada na crítica por meio da reflexão das vivências ao longo de vinte e quatro meses. Os egressos dessa formação são capazes de transformar os cenários da saúde pública no país que apresentam inúmeras discrepâncias.

A humanização, então, colabora para a melhoria dos atendimentos às populações, proporcionando modelos de serviços que abrangem a saúde integral dos indivíduos, também responsabilizando-os quanto ao autocuidado e autonomia na tomada de decisões. Portanto, praticá-la e estimulá-la no cotidiano das unidades da APS é urgente enquanto uma necessidade imprescindível para a concretização de um SUS de qualidade para todos.

\section{REFERÊNCIAS}

BRASIL. Ministério da Saúde. Secretaria de Atenção à Saúde. Departamento de Atenção Básica. Cadernos de Atenção Básica: Acolhimento à Demanda Espontânea, v. 2. Brasília: MS; 2013. 
BRASIL. Ministério da Saúde. Secretaria de Atenção à Saúde. Departamento de Atenção Básica. Política Nacional de Educação Permanente em Saúde: o que se tem a produzir para o seu fortalecimento. Brasília: MS; 2018.

BRASIL. Ministério da Saúde. Humaniza SUS: Documento base para gestores e trabalhadores do SUS. 4 ed. $1^{a}$ reimpressão, Série B, Textos Básicos de Saúde. Secretaria de Atenção à Saúde. Núcleo Técnico da Política Nacional de Humanização Brasília, 2008. Disponível em: https://bit.ly/3wvKn8m. Acesso em: 03 fev. 2021.

BRASIL. Ministério da Saúde. Política Nacional de Atenção Básica. Série E. Legislação em Saúde. Secretaria de Atenção à Saúde. Departamento de Atenção Básica. Brasília, 2012. 110 p. Disponível em: https://bit.ly/3HkfKrJ. Acesso em: 12 jan. 2021.

BRASIL. Ministério da Saúde. Lei n. 11.129, de 30 de junho de 2005. Institui o Programa Nacional de Inclusão de Jovens - ProJovem; cria o Conselho Nacional da Juventude - CNJ e a Secretaria Nacional de Juventude; altera as Leis ${ }^{\circ}$ s 10.683, de 28 de maio de 2003, e 10.429, de 24 de abril de 2002; e dá outras providências. Diário Oficial da União. 2005.

BORDIN. T. et al. Estudo comparativo da satisfação de usuários e profissionais da saúde com o serviço público odontológico. Ciência \& Saúde Coletiva, v. 22, n. 1, p. 151-160, 2017.

BIZERRIL, D. et al. Papel do cirurgião-dentista nas visitas domiciliares: atenção em saúde bucal. Revista Brasileira de Medicina e Comunidade, v. 37, n. 10, p. 1-8, 2015.

COSTA, G. D. et al. Saúde da Família: desafios nos processos de reorientação do modelo assistencial. Revista Brasileira de Enfermagem da USP, v. 69, n. 1, p. 113-118, 2009.

FUNDAÇÃO ESTATAL SAÚDE DA FAMÍLIA. Projeto Político Pedagógico. FESF/SUS. Disponível em: https://bit.ly/3kocfXo. Acesso em: 20 de janeiro de 2020.

GUERRERO P. et al. O acolhimento como boa prática na Atenção Básica a Saúde. Texto Contexto Enfermagem, v. 22, n. 1, p. 132-140, 2013.

MOIMAZ, S. et al. Qualificação do acolhimento nos serviços de saúde bucal. Texto Caderno de Saúde Coletiva, v. 25, n. 1, p. 1-6, 2017. 
MONTEIRO, M. M; FIGUEIREDO, V. P; MACHADO, M. F. A. Formação do vínculo na implantação do Programa Saúde da Família em uma Unidade Básica de Saúde. Revista da Escola de Enfermagem da USP, v. 43, n. 2, p. 358-364, 2009.

DIAS D’DÁVILA. Secretaria Municipal de Saúde de Dias d'Ávila. Plano municipal de saúde. Dias d'Dávila: Secretaria Municipal de Saúde de Dias d’Ávila, 2017.

RAJÃO F.; MARTINS, M. Atenção Domiciliar no Brasil: estudo exploratório sobre a consolidação e uso de serviços no Sistema Único de Saúde. Ciência \& Saúde Coletiva, v. 25, n. 5, p. 1863-1875, 2020.

SALDIVA, P. H; VERAS, M. Gastos públicos com saúde: breve histórico, situação atual e perspectivas futuras. Estudos avançados, v. 32, n. 92, p. 47-71, 2018.

SAVASSI, L. C. M; LAGE, L.; COELHO, F. L. Sistematização de um instrumento de estratificação de risco familiar: Escala de risco familiar de Coelho-Savassi. Journal of Management and Primary Health Care, Rio de Janeiro, v. 3, n. 2, p. 179-185, 2012.

DIAS D’DÁVILA. Secretaria Municipal de Saúde de Dias d'Ávila. Relatório de gestão: ano 2017. Dias d'Dávila: Secretaria Municipal de Saúde de Dias d’Ávila, 2017.

WARMLING, C. M. Acolhimento e acesso de necessidades de saúde bucal e o agir profissional na Atenção Primária à Saúde. Interface, v. 23, p. 1-15, 2019.

ZUGE, E. Humanização nos serviços de saúde. (Trabalho de conclusão de curso). Porto Alegre: Universidade Federal do Rio Grande do Sul; 2012. 